\title{
Manipulation of visual information does not change the accuracy of distance estimation during a blindfolded walking task
}

\section{Sean Commins*, Kelsie McCormack, Erin Callinan, Helen Fitzgerald, Eoin Molloy, Kerrie Young}

Department of Psychology, National University of Ireland Maynooth, Maynooth, Co. Kildare, Ireland

\section{A R T I C L E I N F O}

\section{Article history:}

Available online 26 July 2013

\section{Keywords:}

Blindfold navigation

Distance estimation

Visual

Idiothetic

\begin{abstract}
A B S T R A C T
While humans rely on vision during navigation, they are also competent at navigating non-visually. However, non-visual navigation over large distances is not very accurate and can accumulate error. Currently, it is unclear whether this accumulation of error is due to the visual estimate of the distance or to the locomotor production of the distance. In a series of experiments, using a blindfolded walking test, we examine whether enhancing the visual estimate of the distance to a previously seen target, through environmental enrichment, visual imagery, or repeated exposure would improve the accuracy of blindfold navigation across different distances. We also attempt to decrease the visual estimate in order to see if the opposite effect would occur. Our results would indicate that manipulation of the static visual distance estimate did not change the navigation accuracy to any great extent. The only condition that improved accuracy was repeated exposure to the environment through practice. These results suggest that error observed during blindfold navigation may be due to the locomotor production of the distance, rather than the visual process.
\end{abstract}

(c) 2013 Elsevier B.V. All rights reserved.

\section{Introduction}

Humans are constantly on the move, finding their way to specific locations in the environment. To reach a target both visual and non-visual sources of information can potentially be used. Visual

\footnotetext{
* Corresponding author.

E-mail address: Sean.Commins@nuim.ie (S. Commins).
} 
information consists of static and dynamic cues (Sun, Campos, Young, Chan, \& Ellard, 2004). Static visual cues can include retinal image size, texture, gradient and binocular disparity (Foley, 1980). Dynamic cues include retinal information generated by the observer's self-motion (optic flow) (Gibson, 1950; Lee, 1980; Sun, Carey, \& Goodale, 1992; Warren \& Hannon, 1990), as well as the motion of objects in the environment (Regan \& Hamstra, 1993; Sun \& Frost, 1998). Humans are also quite competent at navigating to a target without visual input. Non-visual inputs are internally generated as a result of one's body movements (Chance, Gaunet, Beall, \& Loomis, 1998; Mittelstaedt \& Mittelstaedt, 2001). This source of information, often referred to as 'idiothetic information' is provided by muscles and joints, motor efferent signals and vestibular information generated as a result of changes in linear or rotational movement velocities (Tversky, 2000).

Having the ability to update one's current position along a tract (path Integration) is essential for an organism to estimate how far it has travelled and how far it has to go. While a number of studies have demonstrated that visual information (via optic flow) can be used to accurately estimate and reproduce traversed distances (Bremmer \& Lappe, 1999; Redlick, Jenkin, \& Harris, 2001), a number of studies comparing blind, blindfolded and/or sighted participants have shown that spatial competence does not necessarily depend on prior visual experience (e.g., Loomis et al., 1993). Indeed, there have been a number of studies that have shown that idiothetic information alone is quite effective at monitoring distances (see Bigel \& Ellard, 2000).

One of the first studies to demonstrate that distance estimation depends on idiothetic information was conducted by Thomson in 1983 by using the blind walking task. This task required participants to view a target briefly in the distance, typically from between 3 and $22 \mathrm{~m}$ away, close their eyes and walk without vision to where they felt the target was located. He found that 'equally impressive is the extent to which excluding vision does not interfere with performance, especially over the earlier parts of an act' (Thomson, 1983, p. 427). His findings suggested that participants were accurate whether vision was available to them or not. His findings also suggested that precision broke down in the region of 9-12 m, which he attributed to a fading of internalized information about the target's position (occurring after a certain time period $\sim 8 \mathrm{~s}$ ) rather than inadequate distance information.

While many studies have shown, similar to Thomson, that blindfolded humans are able to navigate relatively successfully towards a target, his suggestion of a time limiting component has not been replicated (Fukusima, Loomis, \& Da Silva, 1997; Rieser, Ashmead, Talor, \& Youngquist, 1990), with many authors finding that participants become less accurate in their estimation if the distance is increased (Corlett, Patla, \& Williams, 1985; Fukusima et al., 1997; Glasauer, Amorim, Vitte, \& Berthoz, 1994; Loomis, Da Silva, Fujita, \& Fukusima, 1992; Mittelstaedt \& Mittelstaedt, 2001; Rieser et al., 1990; Steenhuis \& Goodale, 1988). Indeed, there is good evidence to suggest that other factors apart from time can contribute to successful non-visual distance estimation. Such factors include step frequency (Durgin, Akagi, Gallistel, \& Haiken, 2009; Durgin \& Gigone, 2007), walking velocity (Mittelstaedt \& Mittelstaedt, 2001) and stored velocity profiles (Berthoz, Israël, Georges-François, Grasso, \& Tsuzuku, 1995). As well as locomotor information, different cognitive factors, including task demands (Ellard \& Shaughnessy, 2003) and confidence (Philbeck, Woods, Arthur, \& Todd, 2008) may also play a role.

If we accept the idea that people, when navigating non-visually, become less accurate as they walk greater distances, then this suggests that there is a gradual breakdown in the representation of distance. One possibility that might offer an explanation for this is the leaky integrator model (Lappe, Jenkin, \& Harris, 2007). According to this model, as the distance increases in proportion to movement, there is a decay in the integrated distance value over the length of movement. In the model there is a 'gain' component that determines how much a specific movement adds to the integrated distance value (Lappe \& Frenz, 2009) and a 'leak rate' which determines how much the integrated distance value decays over distance length. The nice thing about this model is that it allows for the possibility of different gains. For example, there may be separate gains for visual, vestibular and proprioceptive inputs (Lappe \& Frenz, 2009). It may be possible that manipulation of the gain on any of these inputs may change the accuracy of performance. Sun et al. (2004), for example, have shown that distance estimation varied depending on the cues available and the combination of cues (e.g., vision vs vision + locomotion vs blindfold walking) suggesting that different gains may correspond to different sensory cues. While the Lappe et al. model refers to a dynamic visual gain, that is, visual gain obtained during sighted walking (especially optic flow), we ask whether the visual representation of distance, 
obtained initially in the static view of the scene, can also be manipulated and, if so, does this affect subsequent navigation when blindfold. In a series of experiments we attempt to explore this idea by manipulating the static visual distance representation. This is achieved by (i) allowing the visual representation to fade (i.e., by adding a delay before the commencement of the trial, as suggested by Thomson, 1983), (ii) by enhancing the visual environment through the use of a more enriched environment, (iii) by enhancing the visual representation through mental visual imagery, and (iv) by repeated exposure to the environment. We hypothesize that trial delay should reduce the visual distance representation and therefore lead to poorer accuracy during blindfold navigation compared to a non-manipulated control group. While an enhanced visual landscape, mental imagery, and repeated exposure to a visual scene will all lead to an enhanced visual representation, and result in greater accuracy in distance estimation.

\section{Experiment 1: The effect of vision on navigation}

An initial experiment was conducted to confirm previous research findings (Rieser et al., 1990; Elliott, 1986) that people navigating blindfolded are less accurate as the distance between the starting point and target location increases. In addition, we wanted to demonstrate that error accumulates with distance and that this error accumulation is only observed with idiothetic and not visual navigation.

\subsection{Method}

\subsubsection{Participants}

Thirty volunteers participated in this experiment aged between 19 and 52 with a mean age of 26.43. Eighteen were female and 12 were male. Participants were recruited via an ad hoc sampling method of convenience from the student population of the National University of Ireland Maynooth (NUI Maynooth). All participants were in good health with no dizziness, motion sickness or balance-related conditions. All had normal or corrected-to-normal vision.

\subsubsection{Procedure}

The experiment was conducted in a large open field area (the dimensions of the testing area within this field was $13.4 \mathrm{~m} \times 30 \mathrm{~m}$ ) on the north campus of NUI Maynooth. The ground was relatively flat and even, with little or no tactile landmarks. A retractable tape measure was employed to measure the distances being tested. Four distances $(5,10,15$ and $20 \mathrm{~m})$ were pre-measured and marked. Participants were unaware of the range of distances being tested. A clear visible target was placed at the end of the required distance before each trial commenced. The target was moved for each new distance so that only a single distance could be identifiable at any one time.

Before the experiment each participant was briefed and was then randomly assigned to one of two groups: the first was a visual group $(n=10)$. Participants in this group were allowed the full use of their visual system to guide them to the target. Participants in the second group, the blindfolded group $(n=20)$, were required to navigate blindfolded. All participants were initially led to the starting position and were asked to focus on the target until they were comfortable they could estimate its location. A maximum of $30 \mathrm{~s}$ was allowed. When ready to commence walking, those assigned to the blindfolded group were instructed to pull the blindfold down over their eyes. The experimenter checked that the blindfold was properly secured and the participant could not see. Following this, the experimenter said 'start' in order to commence the trial and instructed participants to walk at a normal steady pace.

As participants commenced walking, the experimenter walked slightly behind them to ensure their safety and comfort. Care was taken to stay slightly behind the participant and to stop only when the participant had stopped. This was to eliminate the participant from picking up cues as to the target location from the experimenter's movements or expectations. Once the participant had indicated their final position by saying 'finished', the stopwatch was stopped and the time recorded. The distance from the target to the participant's stopping point was then measured $(\mathrm{cms})$. A spot on the center 
at the top of the right shoe served as the point from which measurements were taken. Following measurements, participants were then led to the next distance (selected randomly) and commenced the next trial. This was repeated until all four distances were completed.

\subsubsection{Statistics}

Distance from target (which we termed as 'constant error') and time taken to reach the target were measured. In addition, we also compared differences in 'functional error', which we calculated as the distance from target (constant error) expressed as a percentage of overall target distance. The data were subjected to a series of statistical analyses using SPSS (version 19.0). A series of mixed between-within subjects analysis of variance (ANOVA) were conducted to establish if there was a significant effect of distance, time or error, with the appropriate use of post-hoc tests. A star based system of significance was used whereby ${ }^{*} p<.05,{ }^{* *} p<.01,{ }^{* * *} p<.001$.

\subsubsection{Ethics}

The experiment was conducted in accordance with the ethical guidelines for research as set out by the Departmental Ethics Committee at NUIM and the Psychology Society of Ireland. Upon volunteering to assist in research, participants were given a briefing and were made aware of the general nature of the study. Each participant was required to read and sign a consent form, detailing that they were taking part on a voluntary basis and that the use of the blindfold may cause disorientation and affect balance. If they had any underlying medical conditions or experienced dizziness, motion sickness or balance problems, which may be aggravated by being blindfolded, they were advised not to participate. Participants were informed that they were free to remove the blindfold at any stage, if they felt uncomfortable. In addition, participants understood that their data would be kept confidential and they were free to withdraw themselves or their data from the experiment at any time. Participants were debriefed upon completion of the experiment, whereupon they were invited to ask questions about the nature of the experiment, and thanked for giving up their time to take part. All data obtained remained confidential and no identities were revealed during the analysis.

\subsection{Results}

Fig. 1a shows that the mean distance from target obtained for the visual group remained relatively constant across the four distances ( $5 \mathrm{~m}: 18.8+/-2.1 \mathrm{~cm} ; 10 \mathrm{~m}: 29.06+/-4.8 \mathrm{~cm} ; 15 \mathrm{~m}: 25.34 \mathrm{~cm}+/-$ $5.1 \mathrm{~cm} ; 20 \mathrm{~m}: 34.41+/-3.7 \mathrm{~cm})$. In comparison to this, the mean values obtained for the blindfolded group increased over the four distances $(5 \mathrm{~m}: 58.7+/-11.7 \mathrm{~cm}$; $10 \mathrm{~m}: 95.5+/-10.2 \mathrm{~cm})$, with the greatest distance from target found at $15 \mathrm{~m}(158.5+/-19.4 \mathrm{~cm})$ and $20 \mathrm{~m}(263.3+/-37 \mathrm{~cm})$. A mixed between-within subjects analysis of variance (ANOVA) was conducted to assess the impact of visual manipulation on participants' mean distance estimation scores across the four distances for both groups. A significant main effect for distance, $F(3,26)=12.732, p=.001$, and group, $F(1,28)=34.925$, $p=.001$, was found. The vision group was more accurate in their estimation compared to the blindfolded group. Furthermore, a significant Distance $\times$ Group interaction was also noted, $F(3,26)=9.041, p=.001$. Bonferroni corrected $t$-tests revealed that participants in the blindfolded group were significantly less accurate at $15 \mathrm{~m}$ and $20 \mathrm{~m}$ compared to $5 \mathrm{~m}$ (both $p<.05$ ) and that they were significantly less accurate at $20 \mathrm{~m}$ compared to $10 \mathrm{~m}(p<.05)$. No differences were noted for the visual group. However, when we compared the functional error, we noted that the mean error across distances for the blindfolded group remained constant $(11.7+/-2.3 \%, 9.5+/-2 \%, 10.5+/-1.3 \%$ and $13.1+/-1.8 \%$ for $5 \mathrm{~m}, 10 \mathrm{~m}, 15 \mathrm{~m}$ and $20 \mathrm{~m}$, respectively) with no significant difference between the four distances $(F(3,57)=.755, p>.05)$.

Fig. $1 \mathrm{~b}$ illustrates the differences between the two experimental groups in the time taken to complete the task at each distance. Participants in the visual group increased the time taken to reach the target across the four distances from $4.4+/-0.28 \mathrm{~s}$ at $5 \mathrm{~m}$ to $14.2+/-0.2 \mathrm{~s}$ at $20 \mathrm{~m}$. Likewise, the time taken for participants in the blindfolded group also increased across the four distances from $5.16+/-$ $0.27 \mathrm{~s}$ at $5 \mathrm{~m}$ to $18.26+/-0.72 \mathrm{~s}$ at $20 \mathrm{~m}$. A mixed between-within subjects ANOVA revealed a significant main effect for distance, $F(3,26)=148.878, p=.001$, and a significant main effect for group, $F(1,28)=20.375, p=.001$, with the vision group reaching the target quicker than the blindfolded 

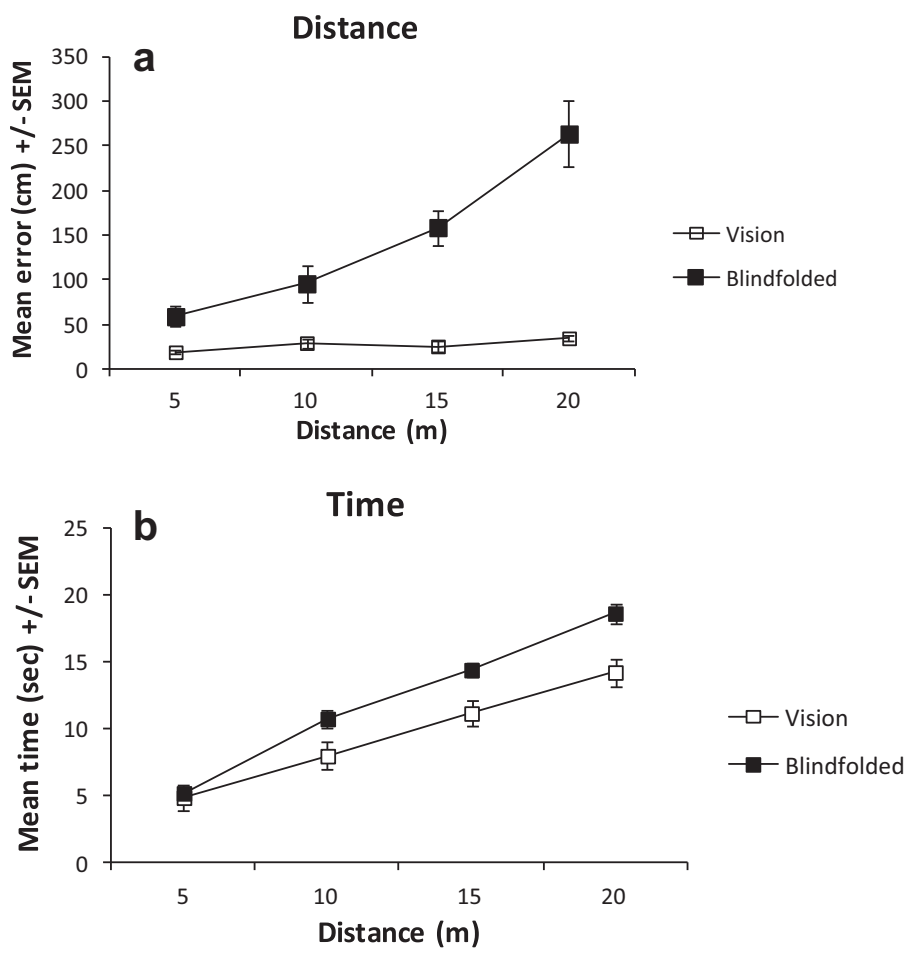

Fig. 1. Effects of vision on accuracy (a) and time (b) of reaching the target, starting from $5,10,15$ and $20 \mathrm{~m}$ away from the target.

group. In addition, a significant Group $\times$ Distance interaction was found, $F(3,26)=5.542, p=.004$. A series of independent $t$-tests were carried out to compare the differences in time between the groups at each distance. There was a significant difference in time found between the two groups at $10 \mathrm{~m}$, $15 \mathrm{~m}$, and $20 \mathrm{~m}(t(26.85)=4.15, p=.001 ; t(26.64)=5.84, p=.001$ and $t(22.26)=5.51, p=.001$, respectively). There was no significant difference found at $5 \mathrm{~m}(t(28)=0.743, p=.464)$. Bonferroni corrected $t$-tests revealed that all distances were significantly different from each other (all $p<.05$ ) for both groups.

These results confirmed that blindfolded navigation becomes increasing less accurate as the distance between the starting position and target location increases. However, when adjusted for distance to be traveled, the error remained constant across the four distances.

\section{Experiment 2: The effect of delay on blindfolded navigation}

Having demonstrated that idiothetic input accumulates a constant error, we had hypothesized that if the visual representation could be manipulated then it may be possible to change the accuracy in blind navigation. One possibility is to introduce a delay into the experiment, whereby the participant observes the target, puts on the blindfold but does not commence navigation until is instructed to do so. As originally suggested by Thomson (1983), the memory of the observed target and imagined environment should decay with time and this, in turn, would influence performance. Although, subsequent research has failed to show such a time effect (Rieser et al., 1990; Elliott, 1986), the delay component in these experiments were either very short (e.g., 2-4 s in Elliott, 1986 and $4 \mathrm{~s}$ in Thomson, 1983) or long (e.g., $30 \mathrm{~s}$ in Elliott, Jones, \& Gray, 1990), thereby allowing for the possibility of mental rehearsal at the short delays or a fading of the representation over the long delays. This experiment will examine delays following $10 \mathrm{~s}$ (see also Rieser et al., 1990). 


\subsection{Method}

Experiment 2 was conducted in the same large open field area on the campus of NUI Maynooth (as above). The same four distances $(5,10,15$ and $20 \mathrm{~m}$ ) were also tested in this experiment. The procedure was identical to that described previously. However, in order to test the effect of a time delay on distance estimation, 36 participants ( 18 males and 18 female, with a mean age of 25 years) were randomly assigned to one of two groups. Participants in Group $1(n=18)$ were initially allowed to observe the target (at a particular distance) for a maximum of $30 \mathrm{~s}$. They were then blindfolded, requested to navigate to each of the four distances and stop when they thought that they had reached each of the targets (blindfolded group). Participants in Group $2(n=18)$ were also required to navigate blindfolded to each distance but when they had put on the blindfold they were requested to delay the start of the trial for 10 seconds before proceeding to navigate to the target (blindfolded+delay group).

\subsection{Results}

Fig. 2a illustrates that that the mean distance from the target obtained by both groups increased with increasing distance. The mean constant error in the blindfolded group increased from $87.8+1-$ $11 \mathrm{~cm}$ at $5 \mathrm{~m}$ to $348.7+/-36 \mathrm{~cm}$ at $20 \mathrm{~m}$. While the blindfolded+delay group accuracy also got worse, from a mean constant error of $118.3+/-17 \mathrm{~cm}$ at $5 \mathrm{~m}$ to $299.8+/-41 \mathrm{~cm}$ at $20 \mathrm{~m}$. A mixed between-
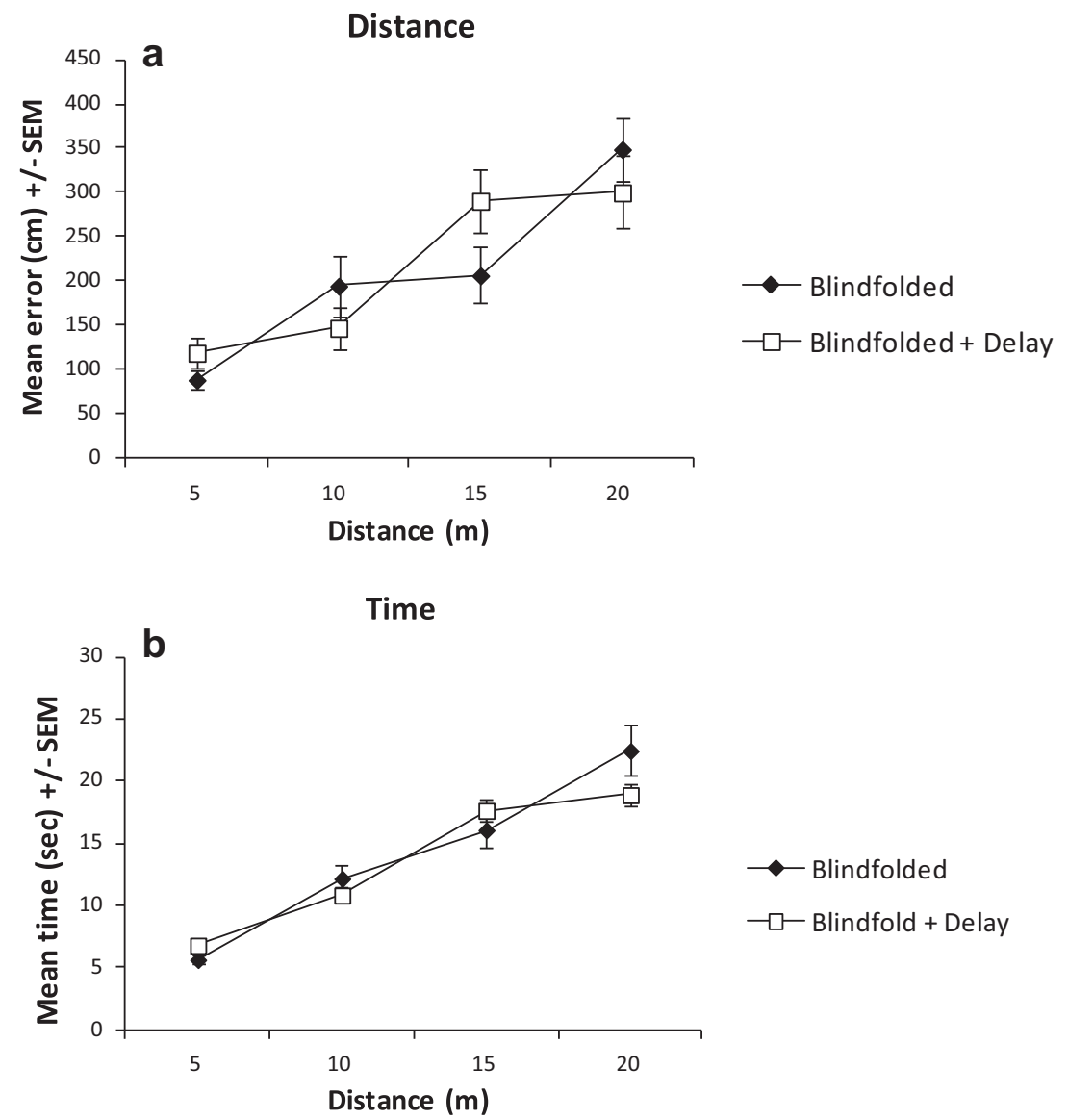

Fig. 2. Effects of delay on accuracy (a) and time (b) of reaching the target, starting from various distances from the target. 
within subjects ANOVA was conducted to compare the two groups across the four distances. A significant main effect for distance, $F(3,32)=27.14, p=.001$, but no significant group effect, $F(1,34)=0.23$, $p=.881$, was found. While an overall significant Distance $\times$ Group interaction, $F(3,32)=5.128, p=.01$, was found, independent $t$-tests revealed no difference between the groups at any of the four distances tested. Bonferroni corrected $t$-tests revealed that all distances were significantly different from each other (all $p<.05$ ). When we examined the functional error no difference between the two groups was found $(17+/-2.3 \%$ for blindfolded group and $18.1+/-2.55$ for the blindfolded+delay group; $F(1,34)=0.196, p=.661$. In addition, there was no effect for distance $(F(3,102)=1.925, p=.130)$, suggesting the error remained the same when controlled for distance travelled.

Analysis for time was then conducted (Fig. $2 \mathrm{~b}$ ) and this revealed a very similar pattern. An overall significant main effect for distance, $F(3,32)=73.6, p=.001$, was found but no significant group effect, $F(1,34)=0.22, p=.642$. An overall significant Distance $\times$ Group interaction, $F(3,32)=4.412, p=.01$, was found, with an independent $t$-test showing that the blindfolded+delay group was significantly slower $(6.8+/-0.4 \mathrm{~s} ; t(34)=2.156, p=.38)$ at reaching the $5 \mathrm{~m}$ target compared to the blindfolded group $(5.6+/-0.3 \mathrm{~s})$. No other differences were noted.

\section{Experiment 3: The effect of environment on blindfolded navigation}

Different sources of visuo-spatial information are available to the navigator, but the type of information depends upon the environment in which the navigator finds itself. For example, if the navigator is in an open field, cues, objects and landmarks within this environment may allow for the determination of the navigator's position and orientation. The relationship among these may then be encoded, which allows for the relatively accurate non-visual navigation. Alternatively, if the navigator is in an enclosed environment, it is the overall geometric shape of the environment, as well as, the cues within this environment that allows for the determination of orientation and position (Cheng \& Newcombe, 2005; Sturz, Kelly, \& Brown, 2010). It has been shown that idiothetic navigation can be sensitive to the knowledge of the environment. Nico, Israël, and Berthoz (2002), for example, found that within a small enclosed environment, blindfolded participants were always shorter in their target estimation, whereas in a larger room, they tended to go further than the target. The authors suggested that the availability of visual information in the smaller environment could provide a better spatial estimation as there are more cues available (distance from walls, overall shape and cues). Furthermore, Kelly, McNamara, Bodenheimer, Carr, and Rieser (2008) suggested that the shape of the environment may also have an effect, finding that angular environments can provide better spatial performance compared to non-angular ones. In this experiment, we wish to examine whether the accumulation of errors observed during non-visual navigation in the open field will be eliminated with navigation within an indoor arena. We suggest that this might be the case due to the great availability of cues within the indoor environment (a visually enriched sports hall), allowing for a greater elaboration of the visual representation. The only landmarks available in the outdoor environment consist of buildings at large distances from the experimental setup.

\subsection{Method}

The procedure was identical to that described previously. However, in order to test the effect of the environment on distance estimation, 30 participants (mean age of 26.9; 11 males and 19 females) were randomly assigned to one of two groups. Participants in Group 1 (blindfolded outdoors; $n=15$ ) were required to walk blindfolded towards the target at four different distances $(5,10,15$, $20 \mathrm{~m}$ ). The location used was the same as that in the pervious experiments (a field on the North campus of NUI Maynooth). The nearest landmark (a building) was located at over $35 \mathrm{~m}$ away. Participants in Group 2 (blindfolded indoors; $n=15$ ) were also required to walk blindfolded to each of the four distances but in this condition the experiment was conducted indoors in a large sports hall. The dimensions of the sports hall were $30 \mathrm{~m} \times 21 \mathrm{~m}$ and contained multiple visual stimuli including basketball hoops on walls, a climbing wall, painted lines on the floor etc. The indoor experiment was conducted at times when the hall was empty and noise free. 

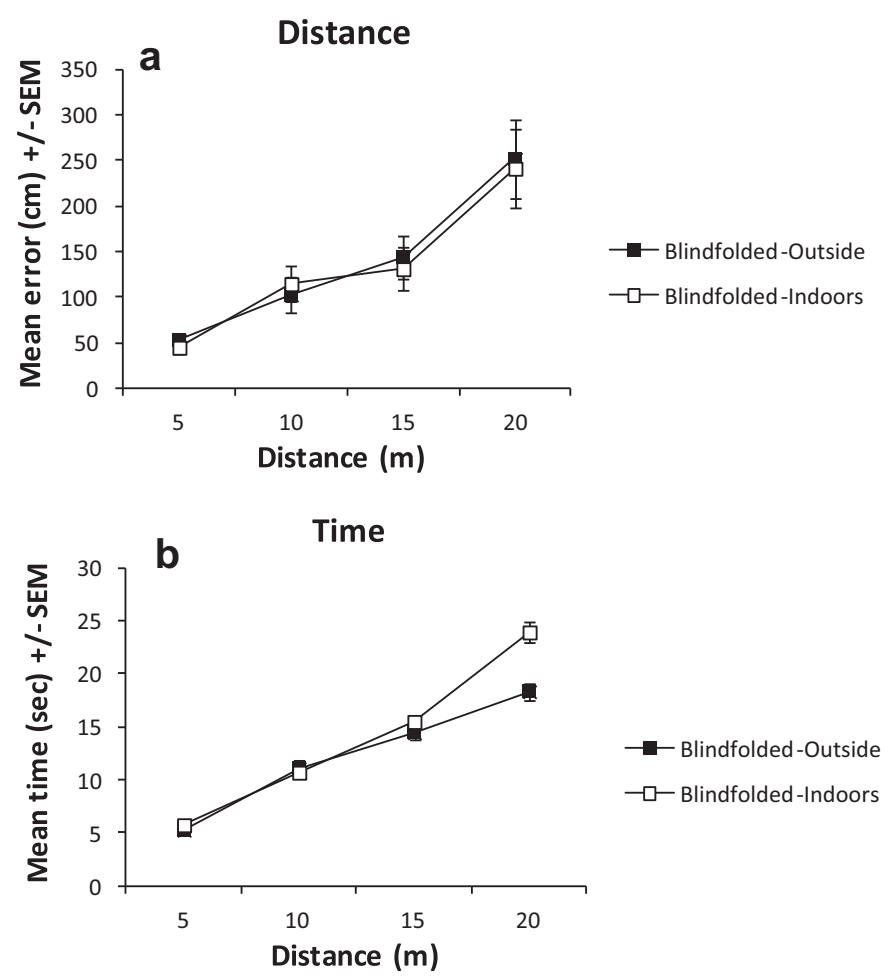

Fig. 3. Effects of location (indoors vs outdoors) on accuracy (a) and time (b) of reaching the target, starting from various distances from the target.

\subsection{Results}

Fig. 3a demonstrates that there was no effect of environment/location on the accuracy of participants. An overall effect for distance was found, $F(3,26)=28.3, p=.001$, but no group, $F(1,28)=0.048, p=.828$, or Distance $\times$ Group interaction, $F(3,26)=0.141, p=.935$. Bonferroni corrected $t$-tests revealed that all distances were significantly different from each other (all $p<.05$ ). When we examined functional error, as with previous experiments, no significant main effect for distance $(F(3,84)=1.275, p=.288)$, group $(F(1,28)=0.069, p=.79)$ or Distance $\times$ Group interaction was found $(F(3,84)=0.218, p=.883)$. Analysis was then conducted for the time taken to reach each target (Fig. 3b) and this revealed a significant effect for distance, $F(3,26)=243.4, p=.001$, group, $F(3,28)=5.04, p=.033$, and Distance $\times$ Group interaction, $F(3,26)=15.4, p=.001$. However, independent $t$-tests revealed that the blindfolded indoor group was significantly slower at $20 \mathrm{~m}$ only; no other group difference were noted. Thus, despite being slower, participants at $20 \mathrm{~m}$ indoors were just as (in)accurate as those performing the task outside. This experiment demonstrates that attempts at manipulating the visual representation by changing the visual environment did not seem to enhance the performance of participants.

\section{Experiment 4: The effect of imagery on blindfolded navigation}

Mental practice has long been demonstrated to not only enhance actual physical performance generally (Jones \& Stuth, 1997), but enhance actual muscular strength (Stevens \& Stoykov, 2003). This remarkable idea has led to an explosion of research and practical applications in fields of rehabilitation (Grangeon et al., 2010), training (Arora et al., 2011) and sport (Munzert, Zentgraf, Stark, \& Vaitl, 2008). Levy, Nicholls, and Polman (2011), for example, showed that both a sportsperson's confidence and 
subsequent performance could be enhanced by mental practice. Despite this, the effect of mental imagery and practice on navigation has not been investigated to any great extent. One study that has attempted to examine the effect of mental simulation on blind navigation was conducted by Vieilledent et al. (2003). These authors demonstrated that mental practice of a hexagonal path produced a more accurate performance than rest alone (i.e., no practice), and performance was equivalent to those that had physically practiced the route. However, this effect was only observed when the distances along the path route remained constant. As visually imaging a scene and visualizing the actual scene produce an overlap in the neural circuitry; about two-thirds of the same brain areas being commonly activated (Kosslyn, Thompson, \& Alpert, 1997), it can be suggested that visual imaging a path may prove to be an effective method by which the visual representation can be manipulated during the blind navigation task. Our fourth experiment aims to test this idea.

\subsection{Methods}

In this experiment participants were required to navigate to 4 distances $(5,10,15,20 \mathrm{~m})$ while blindfolded in the same field as described above. Thirty-four participants (18 male, 16 female and having a mean age of 21.1) were used in this experiment. Participants were randomly assigned to two groups. Participants in Group $1(n=17)$ were initially allowed to observe the target. They were
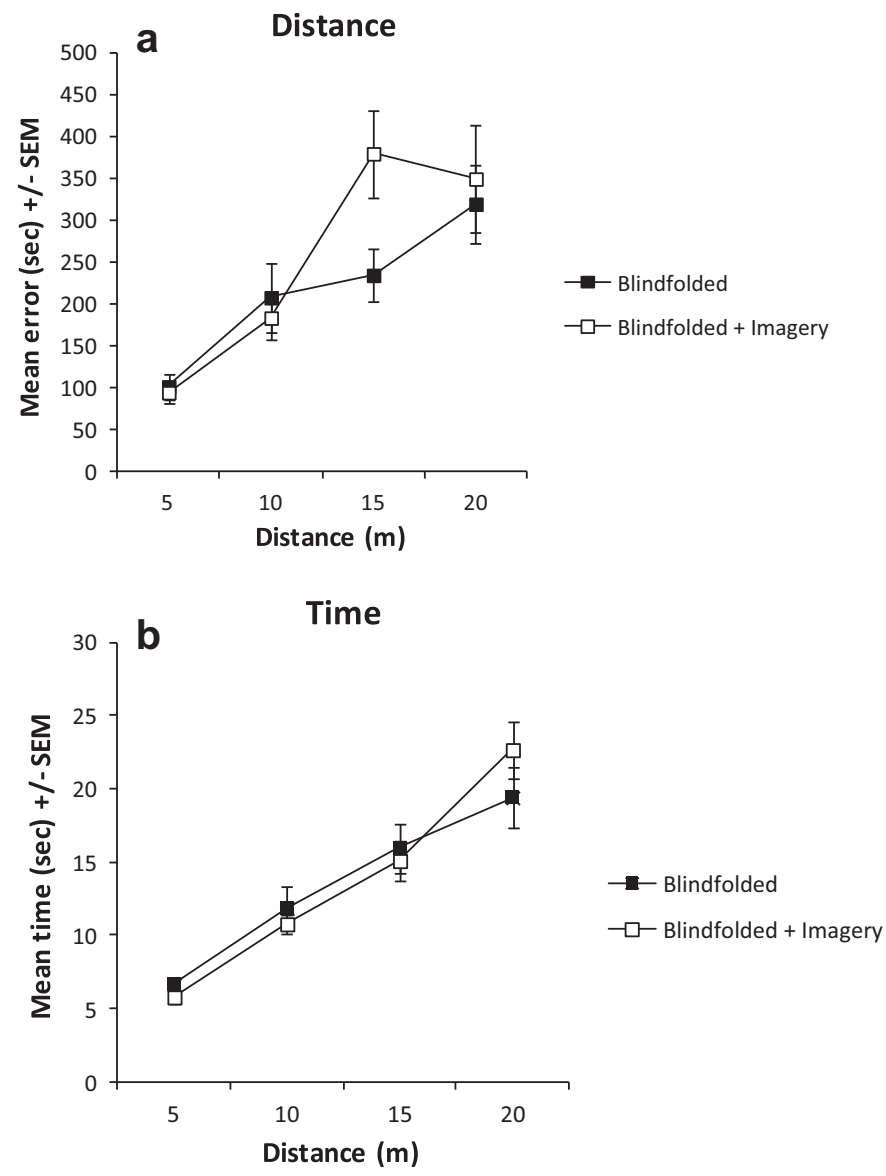

Fig. 4. Effects of imagery on accuracy (a) and time (b) of reaching the target, starting from 5, 10, 15 and $20 \mathrm{~m}$ away from the target. 
then blindfolded, requested to navigate to each of the 4 distances and stop when they thought that they had reached the target (blindfolded group). Participants in Group 2 (blindfolded+imagery, $n=17$ ) were also required to navigate blindfolded to each distance, but before they commenced the trial they were allowed to imagine the distance. To promote this process, the participants were first instructed to observe the target. They were then asked to imagine the distance in their mind (they were also asked to close their eyes). Following this, they were requested to imagine themselves walking the distance. Then, they were asked to open their eyes and observe the target again. Following this, they were requested to close their eyes once more and imagine walking the distance. When this was completed, participants commenced the trial. This procedure was repeated for all 4 distances.

\subsection{Results}

Fig. 4a demonstrates that both the blindfolded and blindfolded+imagery groups increased the mean constant error as they navigated the distances $5 \mathrm{~m}$ to $20 \mathrm{~m}$. A $2 \times 4$ mixed factorial ANOVA was conducted to compare the two groups across the 4 distances. An overall effect was found for distance, $F(3,30)=23.2, p=.001$, but there was no main effect of group $(F(1,32)=1.096, p=.303)$ and no Distance $\times$ Group interaction $(F(3,30)=2.404, p=.087)$. This suggests that imagining the distance and the location of the target, prior to actually walking, did not seem to improve the accuracy in reaching it. When we examined the functional error no difference between the two groups was found $(18.1+/-$ $2.8 \%$ for blindfolded group and $19.9+/-3 \%$ for the blindfolded+imagery group; $(F(1,32)=0.492$, $p=.488$ ). In addition, there was no effect for distance $(F(3,96)=0.687, p=.562)$, suggesting the error remained the same when controlling for distance travelled. In addition, there was no Distance $\times$ Group interaction $(F(3,96)=0.1975, p=.123)$. When we compared the time taken to reach the target (Fig. 4b) an overall significant effect for distance was found $(F(3,30)=42.07, p=.001)$, but there was no effect of group $(F(1,32)=0.007, p=.935)$ and no Distance $\times$ Group interaction $(F(3,30)=1.32, p=.286)$. Our finding would suggest that manipulation of the visual representation by visual mental practice did not improve the accuracy of reaching the target and that the constant error still continued to accumulate as the distance increased. These results are consistent with Corlett, Anton, Kozub, and Tardif (1989) who found no difference in distance estimation between those who were classified as high imagers to those who were low on an imagery questionnaire.

\section{Experiment 5: The effect of practice on blindfolded navigation}

Practice improves performance across many domains, including aspects of blind navigation. For example, Patla, Davies, and Niechwiej (2004) have shown improved performance in obstacle avoidance in blindfolded individuals following practice. In our final experiment we sought to examine the effects of practice on blindfold navigation to determine whether repeated exposure to the same environment can lead to an enhanced accuracy and remove the accumulation of error as the distance to the target increases.

\subsection{Methods}

Thirty-two participants (mean age of 22 years; 16 males and 16 females) were used in this experiment. Participants were randomly assigned to one of two groups. Participants in Group 1 (blindfolded - 1 trial; $n=16$ ) were given 1 trial to walk blindfolded towards the target at the set distances. Participants in Group 2 (blindfolded - 3 trials; $n=16$ ) were also required to walk blindfolded but were given 3 trials at each distance. No feedback was provided between trials. All experiments were conducted outdoors as previously described.

\subsection{Results}

Overall we found no difference in participants' accuracy of the target's location if given 1 trial when compared to 3 trials (Fig. 5a). A mixed factorial ANOVA revealed an overall significant effect for 

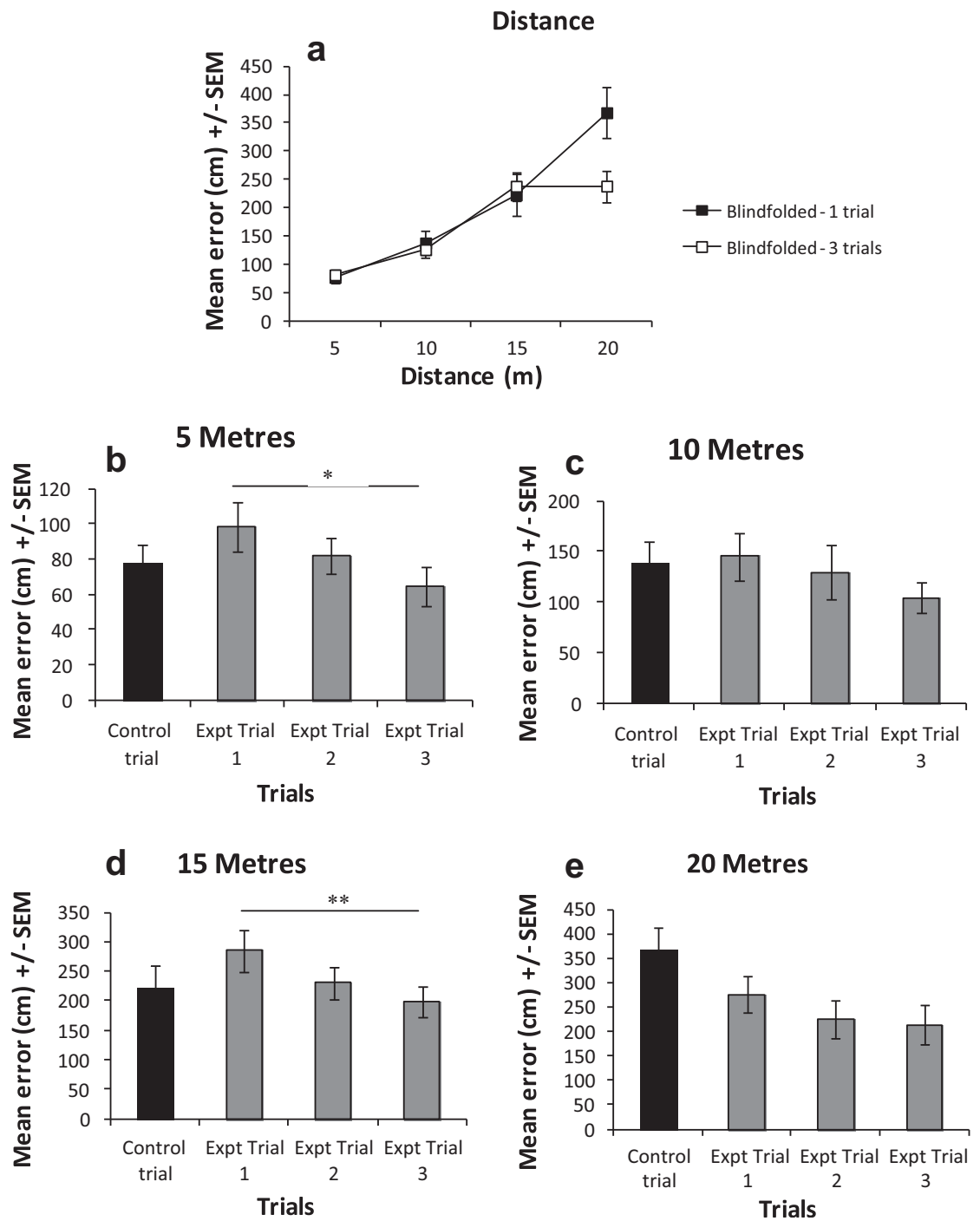

Fig. 5. (a). Effects of practice on accuracy of reaching the target. Bar charts demonstrating how the accuracy changes from trial 1 to trial 3 for $5 \mathrm{~m}$ (b), $10 \mathrm{~m}$ (c), $15 \mathrm{~m}$ (d) and $20 \mathrm{~m}(\mathrm{e}) .{ }^{*} p<.05,{ }^{* *} p<.01$.

distance, $F(3,28)=22.7, p=.001$, with all distances significantly different from each other $(p<.05)$ but no overall significant effect of group $(F(1,30)=1.61, p=.214)$ and no Distance $\times$ Group interaction $(F(3,28)=2.67, p=.067)$. We did, however, find that participants landed closer to the target on 3 compared to trial 1 on distances of $5 \mathrm{~m}$ and $15 \mathrm{~m}(t(15)=2.235, p=.04$ and $t(15)=2.898, p=.01$, respectively) suggesting that there was some effects of learning (Fig. 5b and d). This finding is despite both groups taking a similar length of time to reach their destination (data not shown). This learning effect was further illustrated when we compared the functional error across the three practice trials and the four distances. Although no significant effect for distance was found $(F(3,45)=2.161, p=.106)$, there was a significant trial effect, $F(2,30)=5.485, p=.009$. Bonferroni-corrected $t$-tests revealed that trial 3 had less functional errors $(11.8+/-0.1 \%)$ compared to trial $1(16.6+/-.1 \% ; p=.023)$. No Distance $\times$ Trial interaction was noted $(F(3,90)=0.293, p=.939)$. 


\section{Discussion}

Humans show an impressive ability to navigate without vision, despite being primarily dependent upon this sensory system. Here we demonstrate that humans can navigate blindfolded to a target up to $20 \mathrm{~m}$ away relatively successfully. While this feat has been generally demonstrated previously (Corlett et al., 1985; Fukusima et al., 1997; Glasauer et al., 1994; Loomis et al., 1992; Mittelstaedt \& Mittelstaedt, 2001; Rieser et al., 1990; Steenhuis \& Goodale, 1988), we demonstrate that the accuracy decreased steadily in the blindfolded group as the distance increased, with this group taking longer to complete the task at every distance tested (Experiment 1). Conversely, the visual group remained relatively accurate by comparison, completing the task faster and with lower error scores. While the original findings by Thomson (1983) demonstrated that accuracy broke down in the region of 9-12 m, we did not find this, but observed a systematic increase in error that paralleled the distances to be walked. This is similar to that suggested by Elliott (1986), who reported that accuracy decreased as distance increased and suggested that accuracy was dependent only on the distance that had to be walked. We also show a consistent error pattern that was not significantly different across distances when the overall distance was taken into account.

Our results would indicate that humans have a non-visual odometer that allows for the accurate judgement of distance but this odometer is not perfect, and gains error with increasing distance. Normally, this non-visual system can be easily recalibrated using our visual system (Rieser, Pick, Ashmead, \& Garing, 1995). However, if the system is not re-adjusted the accuracy diminishes, which can lead many navigators to find themselves lost very rapidly, particularly in conditions of poor visibility. What is the source of this error? Currently, it is unclear whether the error is due to the visual estimate of the distance or to the locomotor production of the distance (Durgin et al., 2009). Our experiments (Experiments 2-5) seem to rule out the possibility that the error is due to an estimate of static visual cues. In these experiments we tried to manipulate the visual feedback component through various means and have observed that the non-visual odometer remains unaffected; the error is unaltered and increases with increasing distance. We had hypothesized that by delaying the start of the trial (Experiment 2), that the visual representation would fade (Thomson, 1983) and that this would lead to an increase in error; this, we did not find. Alternatively, we tried to enhance the visual component by using a more enriched environment (Experiment 3 ) or through visual imagery (Experiment 4). Again, we did not see an improvement in performance, as we had hypothesized. These findings would suggest that the source of error is mainly due to the locomotion production of distance, rather than the visual estimation component.

Our findings complement many recent experiments showing that distance error is mainly dominated by various aspects of locomotion. However, there is no consensus as to what aspect of locomotion is deemed the most crucial; velocity, time and stride length are all considered important elements. For example, Mittelstaedt and Mittelstaedt (2001) showed that for all distances, distance estimation depended on walking velocity. However, Durgin and Gigone (2007) argued that locomotor speed is controlled by stride frequency and that people tend to judge themselves as moving faster if they took shorter and more frequent steps. Furthermore, while there is good evidence that rhythmic activity can improve time-keeping, Durgin et al. (2009) also showed that distance estimation is more precise than timing estimation, leading to the conclusion that timing is not a mechanism for distance estimation but rather distance itself or step length is the key component.

Interesting, the only experiment that seemed to show a change in the error was Experiment 5, which looked at practice effects. We had originally thought that repeated exposure to the environment would lead to an enhanced visual representation. We now believe that, as this was the only experiment that contained a manipulation of both locomotion and visual components, the change in accuracy may have resulted from repeated exposure of the locomotor element alone. While we can not rule out the possibility that participants gained greater confidence as the number of blind-walking exposures increased (Philbeck et al., 2008), we suggest the locomotion component can be modified through learning. We suggest that if participants were given more trials, with or without visual feedback between each trial, the accuracy may be improved further. 
In conclusion, while it is possible that any errors that occur during the blind walking task could happen during the processing of visual information, the processing of motor information and/or the process of visuomotor calibration (Sun et al., 2004), our experiments indicate that the different manipulations of the visual representation did not affect subsequent blindfolded navigation. This suggests that the processing of static visual information contributes minimally to the accumulation of error over large distances and that the error observed is mainly due to the locomotion component.

\section{References}

Arora, S., Aggarwal, R., Sirimanna, P., Moran, A., Grantcharov, T., Kneebone, R., et al (2011). Mental practice enhances surgical technical skills: A randomized controlled study. Annals of Surgery, 253, 265-270.

Berthoz, A., Israël, I., Georges-François, P., Grasso, R., \& Tsuzuku, T. (1995). Spatial memory of body linear displacement: What is being stored? Science, 269(5220), 95-98.

Bigel, M. G., \& Ellard, C. G. (2000). The contribution of nonvisual information to simple place navigation and distance estimation: An examination of path integration. Canadian Journal Experimental Psychology, 54, 172-185.

Bremmer, F., \& Lappe, M. (1999). The use of optical velocities for distance discrimination and reproduction during visually simulated self motion. Experimental Brain Research, 127, 33-42.

Chance, S. S., Gaunet, F., Beall, A. C., \& Loomis, J. M. (1998). Locomotion mode affects the updating of objects encountered during travel: The contribution of vestibular and proprioceptive inputs to path integration. Presence-Teleoperators and Virtual Environments, 7, 168-178.

Cheng, K., \& Newcombe, N. S. (2005). Is there a geometric module for spatial orientation? Squaring theory and evidence. Psychonomic Bulletin Review, 12, 1-23.

Corlett, J. T., Anton, J., Kozub, S., \& Tardif, M. (1989). Is locomotor distance estimation guided by visual imagery? Perception and Motor Skills, 69, 1267-1272.

Corlett, J. T., Patla, A. E., \& Williams, J. G. (1985). Locomotor estimation of distance after visual scanning by children and adults. Perception, 14, 257-263.

Durgin, F. H., Akagi, M., Gallistel, C. R., \& Haiken, W. (2009). The precision of locomotor odometry in humans. Experimental Brain Research, 193, 429-436.

Durgin, F. H., \& Gigone, K. (2007). Enhanced optic flow speed discrimination while walking: Contextual tuning of visual coding. Perception, 36, 1465-1475.

Ellard, C. G., \& Shaughnessy, S. C. A. (2003). Comparison of visual and nonvisual sensory inputs to walked distance in a blindwalking task. Perception, 32, 567-578.

Elliott, D. (1986). Continuous visual information may be important after all: A failure to replicate Thomson. Journal Experimental Psychology: Human Percept Performance, 12, 388-391.

Elliott, D., Jones, R., \& Gray, S. (1990). Short-term memory for spatial location in goal-directed locomotion. Bulletin of the Psychonomic Society, 28, 158-160.

Foley, J. M. (1980). Binocular distance perception. Psychological Review, 87, 411-434.

Fukusima, S. S., Loomis, J. M., \& Da Silva, J. A. (1997). Visual perception of egocentric distance as assessed by triangulation. Journal Experimental Psychology: Human Perception and Performance, 23, 86-100.

Gibson, J. J. (1950). The perception of the visual world. Boston, MA: Houghton Mifflin.

Glasauer, S., Amorim, M. A., Vitte, E., \& Berthoz, A. (1994). Goal directed linear locomotion in normal and labyrinthine-defective subjects. Experimental Brain Research, 98, 323-335.

Grangeon, M., Guillot, A., Sancho, P. O., Picot, M., Revol, P., Rode, G., et al (2010). Rehabilitation of the elbow extension with motor imagery in a patient with quadriplegia after tendon transfer. Archives of Physical Medicine and Rehabilitation, 91, 1143-1146.

Jones, L., \& Stuth, G. (1997). The uses of mental imagery in athletics: An overview. Applied and Preventive Psychology, 6, $101-115$.

Kelly, J. W., McNamara, T. P., Bodenheimer, B., Carr, T. H., \& Rieser, J. J. (2008). The shape of human navigation: How environmental geometry is used in maintenance of spatial orientation. Cognition, 109, 281-286.

Kosslyn, S. M., Thompson, W. L., \& Alpert, N. M. (1997). Neural systems shared by visual imagery and visual perception: A positron emission tomography study. Neuroimage, 6, 320-334.

Lappe, M., \& Frenz, H. (2009). Visual estimation of travel distance during walking. Experimental Brain Research, 199, 369-375.

Lappe, M., Jenkin, M., \& Harris, L. R. (2007). Travel distance estimation from visual motion by leaky path integration. Experimental Brain Research, 180, 35-48.

Lee, D. N. (1980). The optic flow field: The foundation of vision. Philosophical Transactions of the Royal Society of London, Series B, 290, 169-179.

Levy, A. R., Nicholls, A. R., \& Polman, R. C. (2011). Pre-competitive confidence, coping, and subjective performance in sport. Scandinavian Journal of Medicine and Science in Sports, 21, 721-729.

Loomis, J. M., Da Silva, J. A., Fujita, N., \& Fukusima, S. S. (1992). Visual space perception and visually directed action. Journal Experimental Psychology: Human Perception and Performance, 18, 906-921.

Loomis, J. M., Klatzky, R. L., Golledge, R. G., Cicinelli, J. G., Pellegrino, J. W., \& Fry, P. A. (1993). Nonvisual navigation by blind and sighted: Assessment of path integration ability. Journal of Experimental Psychology: General, 122, 73-91.

Mittelstaedt, M. L., \& Mittelstaedt, H. (2001). Idiothetic navigation in humans: Estimation of path length. Experimental Brain Research, 13, 318-332.

Munzert, J., Zentgraf, K., Stark, R., \& Vaitl, D. (2008). Neural activation in cognitive motor processes: Comparing motor imagery and observation of gymnastic movements. Experimental Brain Research, 188, 437-444.

Nico, D., Israël, I., \& Berthoz, A. (2002). Interaction of visual and idiothetic information in a path completion task. Experimental Brain Research, 146, 379-382. 
Patla, A. E., Davies, T. C., \& Niechwiej, E. (2004). Obstacle avoidance during locomotion using haptic information in normally sighted humans. Experimental Brain Research, 155, 173-185.

Philbeck, J. W., Woods, A. J., Arthur, J., \& Todd, J. (2008). Progressive locomotor recalibration during blind walking. Perception and Psychophysics, 70, 1459-1470.

Redlick, F. P., Jenkin, M., \& Harris, L. R. (2001). Humans can use optic flow to estimate distance of travel. Vision Research, 41, 213-219.

Regan, D., \& Hamstra, S. J. (1993). Dissociation of discrimination thresholds for time to contact and for rate of angular expansion. Vision Research, 33, 447-462.

Rieser, J. J., Ashmead, D. H., Talor, C. R., \& Youngquist, G. A. (1990). Visual perception and the guidance of locomotion without vision to previously seen targets. Perception, 19, 675-689.

Rieser, J. J., Pick, H. L., Jr., Ashmead, D. H., \& Garing, A. E. (1995). Calibration of human locomotion and models of perceptualmotor organization. Journal Experimental Psychology: Human Perception and Performance, 21, 480-497.

Steenhuis, R. E., \& Goodale, M. A. (1988). The effects of time and distance on accuracy of target-directed locomotion: Does an accurate short-term memory for spatial location exist? Journal Motor Behavior, 20, 399-415.

Stevens, J. A., Stoykov, M. E. (2003). Using motor imagery in the rehabilitation of hemiparesis. Archives of physical medicine and rehabilatation, 84, 1090-1092. Available from http://www.ncbi.nlm.nih.gov/pubmed/12881842.

Sturz, B. R., Kelly, D. M., \& Brown, M. F. (2010). Facilitation of learning spatial relations among locations by visual cues: Generality across spatial configurations. Animal Cognition, 13, 341-349.

Sun, H. J., Campos, J. L., Young, M., Chan, G. S., \& Ellard, C. G. (2004). The contributions of static visual cues, nonvisual cues, and optic flow in distance estimation. Perception, 33, 49-65.

Sun, H. J., Carey, D. P., \& Goodale, M. A. (1992). A mammalian model of optic-flow utilization in the control of locomotion. Experimental Brain Research, 91, 171-175.

Sun, H. J., \& Frost, B. J. (1998). Computation of different optical variables of looming objects in pigeon nucleus rotundus neurons. Nature Neuroscience, 1, 296-303.

Thomson, J. A. (1983). Is continuous visual monitoring necessary in visually guided locomotion? Journal Experimental Psychology: Human Percept Performance, 9, 427-443.

Tversky, B. (2000). Levels and structure of spatial knowledge. In R. Kitchin \& S. Freundschuh (Eds.), Cognitive mapping: Past, present and future (pp. 24-43). London: Routledge.

Vieilledent, S., Kosslyn, S. M., Berthoz, A., Giraudo, M. D. (2003). Does mental simulation of following a path improve navigation performance without vision? Cognitive Brain Research, 16, 238-249. Avalilable from http://www.ncbi.nlm.nih.gov/pubmed/ 12668233.

Warren, W. H., \& Hannon, D. J. (1990). Eye movements and optical flow. Journal of the Optical Society of America, A7, 160-169. 